\title{
Des Architekten liebstes Spiel: Baukunst aus dem Baukasten
}

\section{Matthias Noell}

n der Architekturdiskussion der Moderne nehmen die Vereinfachung

und Systematisierung der architektonischen Bestandteile einen zentralen Platz ein. Nicht selten wurde hierbei auf das Baukastenprinzip als Ideal einer aus wenigen Grundelementen bestehenden Architektur verwiesen. Mit der modularen und dadurch seriellen Architektur wurde einerseits die Hoffnung auf höhere Wirtschaftlichkeit verbunden, angestrebt wurde aber auch eine Klärung des Entwurfsprozesses.

Verfolgt man die Entwicklung des Baukastenspiels seit seiner Entstehung um 1800 und vergleicht sie mit der Geschichte der Architektur in diesem Zeitraum, so zeigen sich zahlreiche Berührungspunkte und Wechselwirkungen, deren Komplexität auf ein grundlegenderes Verhältnis zwischen Spiel und moderner Architektur hinweist. Diese bislang nur punktuell untersuchten Beziehungen von Architektur und Kinderspiel stehen im Mittelpunkt des folgenden Beitrags.

\section{Quadratraster und Bauklotz}

Seit dem späten 18. Jahrhundert zeigten sich in theoretischen Architekturtraktaten Tendenzen zu einer elementaren Analyse der Architektur. In Anlehnung an die Naturwissenschaften versuchten einige Architekturtheoretiker, allen voran Jean-Nicolas-Louis Durand, mit einer Reduktion der entwerferischen Mittel und ihrer systematischen Anwendung allgemeingültige Gesetzmäßigkeiten der Architektur zu de- 


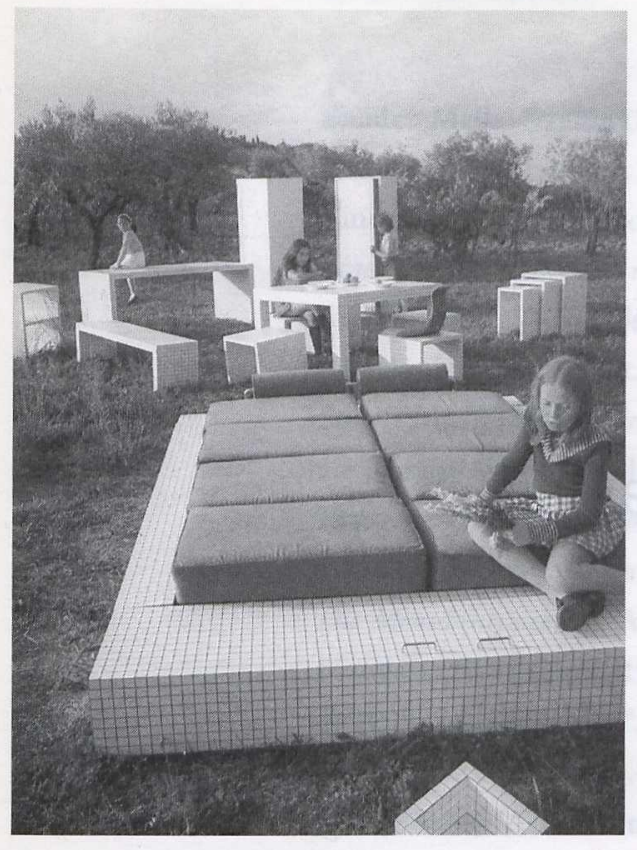

finieren. Eines der wichtigsten Mittel auf diesem Weg war die strikte Modularisierung der Grundbestandteile, die ein harmonisches Zusammenspiel aller Kombinationen und Teilungen ermöglichen sollte. Die frühesten bekannten Baukästen, überliefert durch die Kupferstiche in Bestelmeiers Magazin von 1803, fallen zeitlich mit diesen ersten Elementarisierungsversuchen zusammen. ${ }^{1}$ Im Rahmen seiner Tätigkeit als Professor an der École polytechnique in Paris entwickelte JeanNicolas-Louis Durand eine auf einem Quadratraster basierende Bauentwurfslehre, die er zwischen 1802 und 1805 als Précis des leçons d'architecture publizierte. ${ }^{2}$ Durands Methode garantierte größtmögliche Vielfalt trotz der Anwendung modularer Elemente und zeigte

Abb. 1

1 Bestelmeier (1979), 1. Stück, 5. Platte, Nr. 51 und 3. Stück, 7. Platte, Nr. 388. Für ihre Hinweise danke ich Sibylle Hoiman.

2 Durand (1975).

Die Methode ist u. a bei Vignola oder Robert Morris vorgezeichnet. Zu Durand vgl. v.a. Szambien (1984).

\section{3 „Combinaisons}

borizontales et verticales", vgl. Durand (1975), Bd. 1,

2. Teil, Taf. 20 (Ensembles d'édifices).

4 „Ueber die zweckmäßigste Methode zu projectiren" (1863); vgl. bierzu vor allem Taf. 20 der 1. Auflage des Précis (1802). gleichzeitig die durch ihre systematische Anwendung entstehende Harmonie und Ökonomie auf. Als Konsequenz dieser Methode entstand architektonischer Raum bei Durand ausschließlich aus der Kombination von horizontalen und vertikalen Flächen. ${ }^{3}$ Durands Publikationen wurden vielfach rezipiert und in Fachzeitschriften weiter verbreitet, wie ein Artikel über die zweckmäßigste Methode zu projectiren mit einigen aus Durands Précis übernommenen Schemazeichnungen belegt. ${ }^{4}$

Durands Modularisierung der Architektur und die damit verbundene Rationalisierung ihres Entwurfsprozesses ging wiederum zeitlich einher mit zahlreichen anderen wissenschaftlichen Analysen, die zu „Ur“-Maßen und Grundbestandteilen führen sollten. Die Festlegung auf den ersten „Urmeter" in Paris (1799) vereinte die Fixierung einer Norm mit der Rückführung auf ein Grundmodul, beides notwendige Ausgangspunkte für jeden Standardisierungsprozeß.

Als Friedrich Wilhelm August Fröbel (1782-1852) im Winter 1837/1838 seine ersten Baukästen anfertigen ließ und begann, ihre pädagogische Bedeutung für die Entwicklung des Kindes zu ergründen, war wenige Jahre zuvor, im Jahr 1831, die deutschsprachige Ausgabe von Durands Précis des leçons erschienen. Fröbel entwickelte - als gelernter Feldmesser und Naturwissenschaftler von den Veränderungen der Wissenschaft und Technik nicht unbeeinflußt - ein ganzes System von aufeinander aufbauenden "Spielgaben“. Dritte und vierte "Gabe" bestanden aus 
einem Grundmodul, dem „,in acht Bauklötzchen geteilte[n] Würfel“. 5 Die seit Fröbel verbreiteten Elementarbaukästen zeichneten sich damit vor den sonst in seiner Zeit üblichen Baukästen durch ihre Reduzierung auf wenige grundlegende, neutrale Bauteile und den tiefergehenden pädagogischen Anspruch aus. Ähnlich wie schon bei Jean-NicolasLouis Durand sollte durch Teilung und Kombination die harmonische Beziehung des Einzelnen zum Ganzen gelehrt werden. ${ }^{6}$

In der Anleitung zum „rechten Gebrauch“ des Baukästchens schrieb Fröbel, das Spiel habe auf einem Tisch, „am besten auf einer mit einem Geviert-Netze versehenen Tafel von Holz oder Papier, jede Seite eines Geviertes von der Größe oder Länge einer Breitenkante eines Bauklötzchens“, stattzufinden. ${ }^{7}$ Dieses der Spielfläche zugrundeliegende Achsensystem, das später auch in Nostitz' oder Richters Baukästen übernommen wurde, ähnelte stark dem von Durand in der Lehre an der École polytechnique eingeführten Zeichenpapier mit darauf abgebildetem Quadratraster - dem späteren Millimeterpapier. Die Erfindung dieses Hilfsmittels geht zwar bereits auf die ersten Bauaufmaße 1505/1506 durch Bramante in Rom zurück, und auch als Entwurfshilfe findet es sich bereits vor Durand, z.B. bei Bernardo Antonio Vittone, Philibert de l'Orme, Claude Nicolas Ledoux oder Thomas Jefferson - wenn auch mit vollständig anderen Ergebnissen. Erst auf die Architektur des 20. Jahrhunderts übte diese Entwurfsmethode jedoch auch in formaler Hinsicht maßgeblichen Einfluß aus. Beispielhaft wären J. L. M. Lauweriks, Hendrik Petrus Berlage oder Frank Lloyd Wright, Le Corbusier, Walter Gropius, Max Bill, Oswald Maria Ungers, Peter Eisenman oder Superstudio zu nennen. ${ }^{8}$ Das italienische Architekturbüro Superstudio entwickelte zwischen 1968 und 1969 seine sogenannten istogrammi d'architettura, einen Katalog von aus Würfeln zusammengesetzten, architekturähnlichen Isometrien, bei denen das sichtbare Quadratraster auch zur ästhetischen Grundlage wurde. Ort, Größe und Funktion der Histogramme blieben absichtlich unbestimmt und drückten darin einen universalen Anspruch aus. In einem weiteren Schritt entstanden aus diesen, den Erkenntnisformen Fröbels sehr nahestehenden istogrammi "senza sforzo oggetti mobili, environments, architetture": zwanglos und spielerisch. ${ }^{9}$ Die Möbel der Serie Misura sind bis aufs Äußerste reduzierte Symbole ihrer selbst, vollständig überzogen mit einem feinen Quadratraster, ihrem Ursprung. Der Tisch
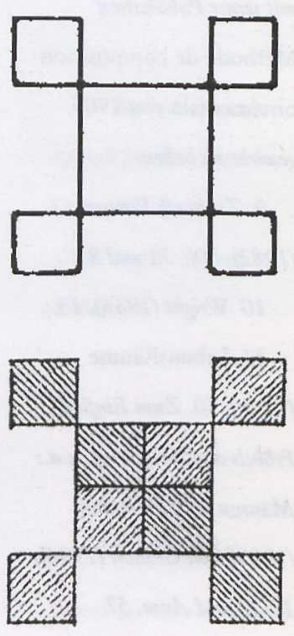

Abb. 2-5

5 Fröbel (1982), 95.

6 Zur Teilung bei

Fröbel vgl. Fröbel (1974),

120-161, bes. 158 (Tafel).

Zur Teilung bei Durand vgl. Durand (1975),

Bd. 1, 2. Teil, Taf. 20

(Ensembles d'édifices).

7 Fröbel (1982), 96. Einerseits ist er einer der Tische, die mit den Bauklötzen - laut Fröbels 


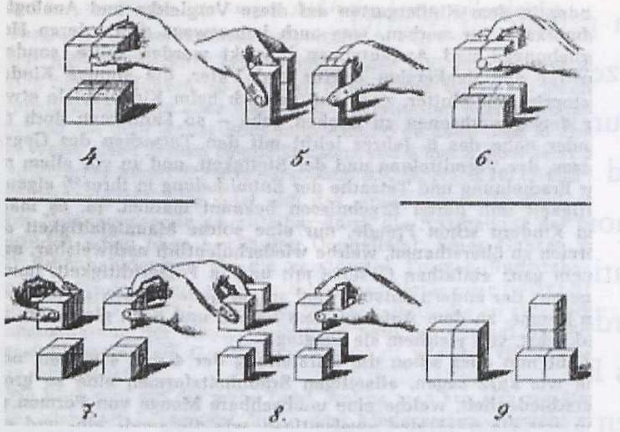

Anleitung zur vierten Gabe - nachgebaut werden sollten, andererseits ist er selbst das dem Spiel zugrundeliegende System, der Tisch mit dem darauf abgebildeten Quadratraster. Er ist aus dem Spiel hervorgegangen, dessen Grundlage er darstellt. Für die komplette Serie Misura wurde unter anderem mit einem Foto geworben, das die Möbel im Freiluft-Showroom im Chianti zeigt, auf der Wiese verteilt, mit Obstbäumen im

Abb. 6

8 Vermittelnd scheint auch Eugène Grasset mit seiner Publikation Méthode de composition ornementale von 1905 gezvirkt zu haben.

9 Zit. nach Pettena (1982), 50f., 73 und 85.

10 Wright (1966), 15.

11 LebensRäume (1999), 50. Zum Einfluß Fröbels auf Wright vgl. u.a.: Manson (1953), Rubin (1989) und Cronon (1994), 15-20 und Anm. 57.

12 Zit. nach Hildebrandt (1904), XIV.
Hintergrund. In diesem Fröbelschen Kindergarten spielen einige Kinder mit und an den Möbeln.

Daß die strengen Spielvorgaben Fröbels in der Praxis tatsächlich befolgt wurden, zeigt die Äußerung des Architekten und Bewunderers der Fröbelschen Baukästen Frank Lloyd Wright: „[...] mehrere Jahre saß ich an dem kleinen Kindergartentisch, über den sich im Abstand von zehn Zentimetern Längs- und Querlinien zogen, so daß lauter ZehnZentimeter-Quadrate entstanden “. ${ }^{10}$ Auf diesem Raster wurde dann gebaut, und es scheint nur noch ein kleiner Schritt zu der Umsetzung der Fröbelschen und Durandschen Vorgaben in die reale Architektur des amerikanischen Architekten, von der die kunsthistorische Forschung unter anderem schreibt: „Das Architektursystem des Amerikaners Frank Lloyd Wright basiert auf einer begrenzten Anzahl architektonischer Bausteine, die sich in ihrer Anordnung unendlich oft variieren lassen. " 11

Fröbels Gestaltungsvorgaben finden sich aber auch direkt in der Entwurfspraxis mancher Architekten wieder, wie ein Vergleich der Illustrationen Fröbels und Peter Eisenmans deutlich macht. Eisenmans House III von 1969-1971 entstand aus der Zerlegung eines Würfels in siebenundzwanzig kleinere Würfel und deren anschließender Drehung und Neupositionierung.

\section{Architekt und Baukasten}

Die Verbindung von Baukästen und Architekten scheint zunächst eine logische Folge des lernenden Kindes und der Entdeckung und Förderung seiner Begabungen zu sein, die bereits Plato in seiner seit Paul Hildebrandt vielzitierten Äußerung erwähnte: „Einer, der späterhin [...] ein guter Baumeister werden soll, muß schon im Kinderspiel kleine Häuslein aufsetzen [...]. [...] Ein künftiger Architekt z.B. muß mit Maß und Richtschnur umgehen lernen. ${ }^{\text {"12 }}$ Allerdings spielten sicherlich 
mehr Kinder mit Baukästen als Architekten ausgebildet wurden, und zudem übte nicht jeder gute Architekt als Kind mit einem Baukasten.

Auch ist eine direkte Verbindung zwischen der Elementarlehre an den neu entstehenden polytechnischen Hochschulen und derjenigen im Kindergarten bislang nicht nachzuweisen. Zwischen Durand und Fröbel verlief trotz aller Gemeinsamkeiten die Grenze zweier Berufe.

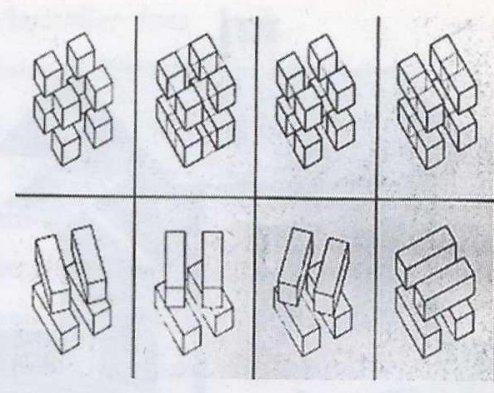

Abb. 7 Jedoch ist seit der Mitte des 19. Jahrhunderts eine Vermischung der Sphären des Spiels und der realen Architektur feststellbar, und das jenseits einer direkten Einflußnahme der Architektur auf das Spielmittel. Zwei Jahre nach dem Beginn der Vollendung des Kölner Doms im Jahr 1842 bemerkte ein Rezensent eines neuen französischen Elementarbaukastens: „[...] und es ist eine Kleinigkeit, den cölner Dom und andere Gebäude, die nicht fertig werden wollen, mittelst der darin befindlichen Blöcke zu schneller Vollendung zu bringen. ${ }^{\text {"13 }}$ Dem Bauunternehmen, zu dem man wohl keine französischen Steine benötige, wie dem Menschen überhaupt attestierte der Rezensent zwischen den Zeilen einen kindischen Zug: Denn „besonders beim Bauspiele werden sich die Erwachsenen immer als große Kinder zeigen. ${ }^{\text {" } 14}$

Nach den ersten Systematisierungsversuchen von Friedrich Fröbel sowie der Gründung der ersten Baukastenfabrik im Jahr 1850 setzte eine Weiterentwicklung der Baukästen ein, die häufig auf Architekten zurückging. Zusammen mit seinem Bruder, dem Erfinder und Flugpionier Otto Lilienthal, begann Gustav Lilienthal in den 1870er Jahren mit ersten Bauklötzen aus Kunststein zu experimentieren. Als Architekt war er unzufrieden mit den realitätsfernen und abstrakten Holzklötzen Fröbels, die Kinder sollten mit einem neuen Material zur Nachahmung der bestehenden Architektur angeleitet werden. Seine Erfindung verkaufte Lilienthal 1879 nach fehlgeschlagenen Vermarktungsversuchen an Friedrich Adolf Richter, der mit größerem unternehmerischen Geschick den Anker-Steinbaukasten weltweit bekannt machte. Neben den hervorragend aufeinander abgestimmten Bausteinen zeichnete Lilienthals Erfindung die Einführung von Vorlagenheften aus, die neben Kirchenbauten auch Mausoleen, Denkmäler oder Burgen bereithielten. Gustav Lilienthal mußte sich aus dem Baukastengeschäft zurückziehen und widmete sich zunächst wieder seinem eigentlichen Beruf, der Architektur. In Lichterfelde bei Berlin konnte er in den 1890er Jahren mehr als dreißig Landhäuser errichten, die vielfach im stilistischen Kleid

13 Reproduktion des Artikels in: Noschkal Knerr (1986), 28. 14 Noscbka/Kerr (1986), 28. 

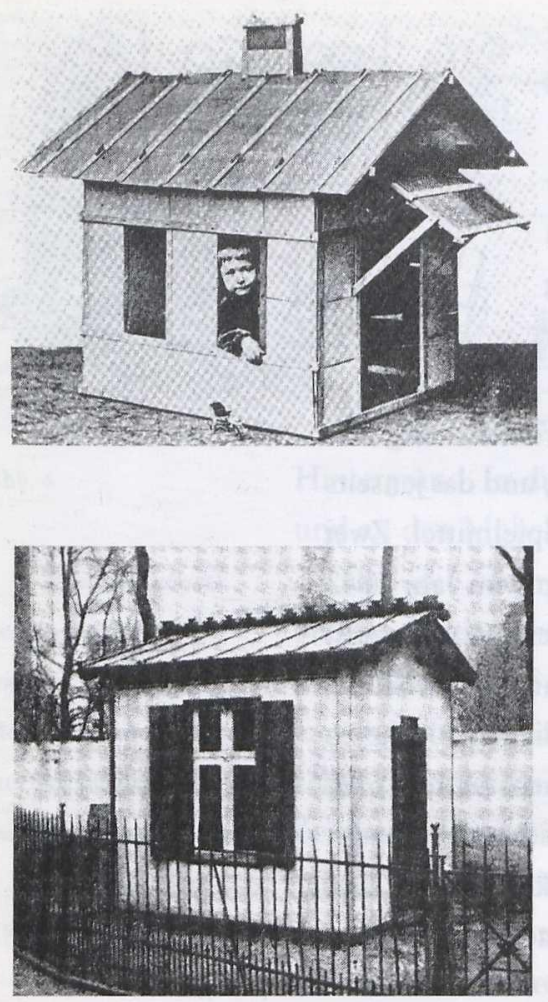

kleiner Burgen mit Zugbrücken und zinnenbekrönten Türmen auftreten. ${ }^{15}$ Man muß jedoch nicht auf die Nähe der burgartigen Villen zu den Burgen der Bau-kästen verweisen, um eine Verbindung zwischen Baukasten und Baukunst festzustellen. Eine unmittelbare Beziehung besteht zwischen der Produktion von Kunststeinen für Baukästen und der nur unwesentlich später erfolgten Herstellung von Zementhohlsteinen. Diese sogenannten Terrast-Steine entwickelte Lilienthal für die von ihm mitbegründete Siedlergenossenschaft Freie Scholle. Ähnliche Experimente mit seriell vorgefertigten Baumaterialien ziehen sich als Leitmotiv durch die moderne Architektur des 20. Jahrhunderts. Nebenbei erfand Gustav Lilienthal weitere wegweisende Bau- und Konstruktionsspiele, unter anderem zwischen 1880 und 1890 eines aus gelochten und mit Splinten und Keilen verbundenen Leisten, aus dem man z.B. ein großes, bewohnbares Spielzeughaus für Kinder zusammensetzen konnte. Eine Weiterentwicklung seiner Terraststeine führte auch $\mathrm{zu}$ einem ganzen System aus vorgefertigten und zerlegbaren Terrasthäusern, die er seit etwa 1900 als Baracken, Gartenhäuschen und sogar als

15 Schäche (1989), 91-118.

16 Katalog der TerrastBangesellschaft, abgedruckt in: Schäche (1989), 113.

17 Lilienthal (1930), 97. Wohnhäuser anbieten konnte: „Diese Bauten bieten den großen Vorteil, daß sie ohne an Wohnlichkeit einzubüßen und ohne Zerstörung der Teile zerlegt und anderweitig wieder aufgebaut werden können. "16 Die konstruktive wie formale Nähe des kleinsten der im Katalog angebotenen Häuser, eines einräumigen Pförtnerhäuschens, zu dem Kinderhaus aus Papptafeln und -leisten ist nicht zu übersehen, ein Umstand, der auch den Lilienthals bewußt war: „Die Herstellung war dem Prinzip des Modellbaukastens etwas verwandt. "17 Tatsächlich gab es dieses Haus schließlich in mehreren Größen, im Baukastenformat, als Kinderhaus sowie als reales Gebäude.

Die folgenden Jahrzehnte der Baukastenentwicklung spiegeln die sich ändernde Wahrnehmung von Architektur und Stadt in einer zuweilen bemerkenswerten Schnelligkeit wider. Der Landhausmode, die in Hermann Muthesius' dreibändiger Publikation Das englische Haus von 19041905 ihren Niederschlag fand, folgte zum Beispiel schon 1905 Carl Brandts Kasten Villa Nova, der „zum Bau reizender Landhäuser in modernem Stil“ aufforderte. Mit den handwerklichen Bestrebungen 
der Reformbewegung um 1900 im Einklang, wies der Hersteller hier zudem auf die „gediegene Ausführung“ hin. ${ }^{18} \mathrm{Zu}$ dem Neuen Haus gab 18 Für die folgenden es, auch hierin den Bestrebungen der Landhausbewegung folgend, den zugehörigen Gartenbaukasten, eine bislang noch nicht näher untersuchte Gattung der Bauspiele. Der Entdeckung des Stadt-Ensembles sowie ihren Folgen, den neuen Disziplinen des Städtebaus und der Städtebaugeschichte mit ihren jeweiligen Initialpublikationen - Camillo Sittes Der Städtebau nach seinen künstlerischen Grundsätzen (1889, 1908 in vierter Auflage erstmals mit Städte-Fotografien erschienen) und Adolf Erich Brinckmanns Deutsche Stadtbaukunst in der Vergangenbeit (1911) - antworteten die Hersteller mit P. F. Messerschmitts „Alte Stadt“ (1904), Brandt's Städtebaukasten (1911) oder dem Schweizerstädtchen (ca. 1920) eines Mitglieds des Schweizer Werkbundes, Carl Zweifel. Die Ideale der Heimatschutzbewegung und des aufkommenden Denkmalschutzes finden sich ebenfalls in den genannten Kästen wieder, wie auch in der Heimatliche[n] Baukunst für Stadt und Land (1910), dem Dorfbaukasten "Heimatkunst" (vor 1912), dem Künstler-Modell-Baukasten "Liebe Heimat" (1916) oder dem Kasten Altes Stadtbild (1925). Auch der Pädagoge und Spielzeughistoriker Paul Hildebrandt gab sich in seinem Standardwerk über das Kinderspielzeug als der Heimatschutzbewegung nahestehender Zeitgenosse zu erkennen:

Moderne Großstadtkinder, die auf Reisen in eine altertümliche Stadt kommen, pflegen oft genug über die alten, ibnen bäßlich und baufällig erscheinenden Häuser sich lustig zu machen, anstatt sich an der architektonischen Schönheit und am Malerischen dieser Bauten und Straßen zu erfreuen. Haben sie aber zu Hause mit ibrem Baukasten solch ein schönes altes Rathaus selbst schon einmal gebaut, dann werden sie, wenn sie nun ein solches Bawwerk in Wirklichkeit sehen, da es ihnen in allen Details vertraut ist, es nicht belächeln, sie zerden es sich im Gegenteil ganz genau ansehen und werden es bewundern, schätzen und lieben, und Reisen grade durch solche altertümliche Städte wird für sie zu einem reichen Genusse und zu einer schönen und auch künstlerischen Erinnerung werden. ${ }^{19}$

Die gelenkte Erziehung der Kinder durch das Spiel ist auch den Baukästen der DDR abzulesen. Passend zum größten Bauvorhaben des noch jungen Staates, der Stalin-Allee (1951-1960), kam 1953 der Kasten Wir bauen auf! mit nahezu exakten Nachbildungen der projektierten Bauten auf den Markt. Der 1956 erschienene, gleichartige Kasten Neue

Baukästen und Zitate vgl. den Abdruck des Kataloges der Stuttgarter Spiehwarenfirma

Hermann Kurtz, in: Noschka/Knerr (1986), 30 und Leinweber (1999), 237. 19 Hildebrandt (1979), 12. 
Baukunst warb mit einer Abbildung des Hochhauses von Hermann Henselmann am Strausberger Platz, Berlin, und thematisierte mit seinem Namen die geplante Erneuerung der Baukunst unter dem Einfluß Stalins. Stalin war jedoch bereits 1953 gestorben, und in der Folge wurde 1955 auch in der DDR die Wende zum industriellen Bauen vollzogen, der schon im selben Jahr Der kleine Baumeister Rechnung trug. Mit diesem Baukasten und den späteren Kästen Moderner Großblock Baukasten (1959) und Der Kleine Großblock Baumeister (Marke Plaspi, 1970er Jahre) vollzog das ostdeutsche Bauspiel ebenfalls den Schritt zum Plattenbau. Natürlich war neben Wohnungsbaukästen für die Magistrale und dem Wobnkomplex auch ein Bausatz für den geforderten Zentralen Platz zu haben: Palast der Republik. Ein Souvenir aus Berlin aus der Kunststoff-Steck-Serie Formo.

In den zwanziger Jahren fand das Umfeld der modernen Großstadtkinder Paul Hildebrandts seinen Platz im Baukastensortiment. Geschäftshäuser und Siedlungen, vor allem aber das Hochhaus, die beliebteste, wenn auch in Europa seltener ausgeführte Bauaufgabe der Moderne, bildeten sich im Kinderspiel ab. Der Kasten Wolkenkratzer von Georg Kade aus der Niederlausitz entsprach z.B. sehr genau den Ergebnissen des Ideenwettbewerbs Hochbaus am Bahnhof Friedrichstraße von 1921/1922, während Dorando - domus stabilis, als Metall-Stein-Baukasten nicht überraschend, eher den in Stahlskelettbauweise errichteten amerikanischen Vorbildern wie dem New Yorker Woolworth-Building nachempfunden war. Unter weiteren Kästen (Wokra, der Wolkenkratzer-Kasten von Carl Brandt aus dem Jahr 1924 wäre hier beispielsweise zu nennen) stach ein System besonders hervor: Die Ingenius-Holzklötze waren mit einem Nut- und Federsystem versehen, das eine Bauhöhe von etwa sechs Metern ermöglichte und damit alle bisherigen Maßstäbe sprengte. Besonders der größte Baukasten dieser Serie, The New City, warb mit Wolkenkratzern und einer amerikanischen Stadt auf dem Deckel und kann als der stilistisch modernste der zur Verfügung stehenden Architektur-Kästen gelten. Entwickelt wurde Ingenius von einem Professor der Düsseldorfer Kunstgewerbeschule, Wilhelm Kreis, jenem äußerst erfolgreichen Architekten der Bismarcktürme und Monumentalbauten, der sich zwischen 1895 und 1955 mit jedem der politischen Systeme in Deutschland anzufreunden wußte. Kreis' Düsseldorfer Wilhelm-MarxHaus (1922-1924) zählt zu den ersten Hochhäusern in Deutschland und entstand zeitgleich mit Ingenius. Sein Patensohn erinnerte sich später an die erste Begegnung mit dem neu erfundenen Bauspiel: 
Wilhelm hatte mit August Jüngst ein Wunderwerk eines technischen Baukastens entwickelt, baute vor mir Kunstwerke der Architektur mit leichter Hand auf, baute ab und sprach: „Nun Du“. Er sab mir zu, seine Miene wurde traurig, zu meinem Vater sagte er: „Technisch boffnungslos - laß ibn Pbilosoph werden", packte den Baukasten ein und entschreand mit ibm. ${ }^{20}$

20 Arntz (1994),

Ein vergleichbares System für die Großarchitektur entstand erst 70 Jahre 208.

$21 \mathrm{Vgl}$. Speidel

(1995), 179 u. 184.

Dort auch zu Hermann

Finsterlins Stilspiel von 1921.

22 Einer von Paul

Es gab aber durchaus Architekten, die den Zusammenhang zwischen Spiel und Architektur enger gefaßt sehen wollten und die Wechselwirkungen als komplexer ansahen als der an theoretischen, sozialen und politischen Zusammenhängen gänzlich desinteressierte Wilhelm Kreis. Am weitesten ging hier vielleicht Bruno Taut. Sein Baukasten aus farbigen Glaselementen von 1920/1921 offenbart eine wesentliche Seite seiner Architekturauffassung jener Zeit. ${ }^{21}$ Seit der Begegnung des Architekten mit dem Schriftsteller Paul Scheerbart nahm das Glas als symbolhaftes Material eine zentrale Stellung im architektonischen Denken von Bruno Taut ein. Es stand für die Überwindung der althergebrachten, abgeschlossenen Architektur und der mit ihr verbundenen Gesellschaft. Glas stand für Licht und für Farbe, die Taut in den Jahren vor dem Ersten Weltkrieg zu einem weiteren Hauptgestaltungselement seiner Architektur gemacht hatte. Die Urform des Glases, der Kristall, konnte mit seinen geometrischen Grundformen und der ihm eigenen Fähigkeit zur Lichtbrechung als natürliches Vorbild zu Tauts Elementarspiel dienen. Paul Scheerbarts Buch Glasarchitektur und Tauts Glashaus auf der Kölner Werkbundausstellung, beide aus dem Jahr 1914, verwiesen aufeinander und beschworen eine bessere Zukunft: „Ohne einen Glaspalast / Ist das Leben eine Last. “22 Die wichtigste Aufgabe des Architekten sei es, schrieb Taut am 5. Oktober 1920 unter seinem Decknamen Glas an die übrigen Mitglieder der Gläsernen Kette, „das Bedürfnis zum Bauen zu wecken. ${ }^{\text {23 }}$ Die Einsicht in die Notwendigkeit des Bauens sowie die pädagogische Absicht, das Bedürfnis zum Bauen zu wecken, ist in dem kleinen, farbigen Glasbauspiel enthalten. ${ }^{24}$ Sein Glasbaukasten verbindet den Architekten Taut mit dem Kristallographen und Pädagogen Friedrich Fröbel, der einhundert Jahre früher diesbezügliche naturwissenschaftliche 


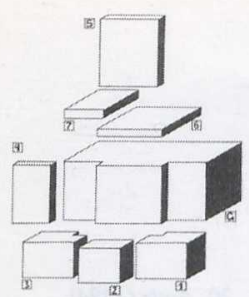

Abb. 10-11

25 Fröbel in einem Brief an Karl Christoph Friedrich Krause vom 24.3.1828, in: Fröbel (1968), 287, Anm. 27. Fröbel war 1812-1816 Assistent des Mineralogen und Kristallographen. Christian Samuel Weiß in Berlin.

26 Walter Gropius in dem unpublizierten Manuskript Wohnmaschinen vom 6.2.1922, zit. nach Nerdinger (1996), 15.

27 Staatliches Baubaus Weimar (1923), 167, Abb. 109; Gropius (1926-1927), 25-30.
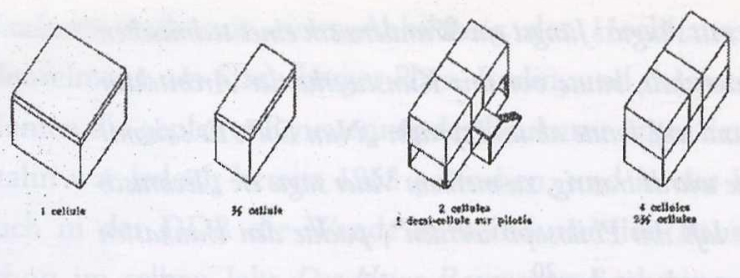

III] \&enètr

Erkenntnisse auf den Menschen übertragen hatte: „Die Kristallwelt verkündigte mir laut und unzweideutig in klarer fester Gestaltung das Leben und die Lebensgesetze des Menschen. ${ }^{\text {“25 }}$

Walter Gropius, der an der von Bruno Taut ins Leben gerufenen Gläsernen Kette beteiligt gewesen war, wurde auf ganz andere Weise von dem Gedanken des Baukastens geleitet. Schon im Jahr 1910 hatte er sich um die Entwicklung standardisierter und genormter Bauteile im Haus- und Wohnungsbau gekümmert. Am neu gegründeten Bauhaus in Weimar schloß er 1922 an diese Gedanken an und propagierte „[...] ein Haus, das aus variablen Versatzstücken, die auf Vorrat hergestellt werden und kombinativ zusammensetzbar sind, etwa in der Art eines AnkerSteinkastens im Großen, zusammengebaut wäre. ${ }^{\text {26 }}$ Gropius entwickelte zusammen mit Fred Forbát im selben Jahr den sogenannten Wabenbau, an dessen Kernbau weitere Räume oder Raumteile angelagert werden konnten. Mit diesem System wurden, im Gegensatz zu den einzelnen Bausteinen Lilienthals, ganze Räume zu Bauklötzen erklärt und miteinander kombiniert - das Prinzip hieß „Vereinigung größtmöglicher Typisierung mit größtmöglicher Variabilität" ${ }^{27}$ Als systematische Weiterentwicklung entstand wenig später der schon im Titel an das Kinderspiel erinnernde Baukasten im Großen.

Auch Le Corbusier war seit 1915 an diesen Themen interessiert. Sein System Dom-Ino bestand aus einem Betonskelett mit zwischenliegenden Bodenplatten. Der dem Tischspiel entlehnte Name deutete - unter zusätzlichem Verweis auf die Bauaufgabe domus - einerseits auf die im Grundriß zu Tage tretenden elementaren Bestandteile Fläche und Punkt hin, andererseits auf die Möglichkeit horizontaler Kombinationen. Die einheitlichen Paßflächen sollten eine einfache Reihung gleicher Häuser zulassen, ein Gedanke, der Le Corbusier auch zur Erfindung des Anbaumöbels und $\mathrm{zu}$ den steckmodulartigen immeubles-villas führte. 1925 konnte er schließlich für den Industriellen Frugès eine Siedlung in Bordeaux-Pessac ausführen. Die maison standardisée in ihren vielfältigen Kombinationsmöglichkeiten geht von einer quadratischen Grundzelle aus, die um eine beliebige Anzahl weiterer Grund- oder Halbzellen erweiterbar ist. 
In der Suche nach dem standardisierten Haus sah Le Corbusier einen der Architektur innewohnenden spielerischen Ernst:

Lorsqu'un standart [sic] est établi, le jeu de concurrence immédiate et violente s'exerce. C'est le match; pour gagner, il faut faire mieux que l'adversaire dans toutes les parties, dans la ligne d'ensemble et dans tous les détails. C'est alors l'étude poussée des parties. Progrès. ${ }^{28}$

Johan Huizinga brachte es in seinem grundlegenden Werk über die Spielkultur des Menschen auf folgende, nicht unähnliche Formel:

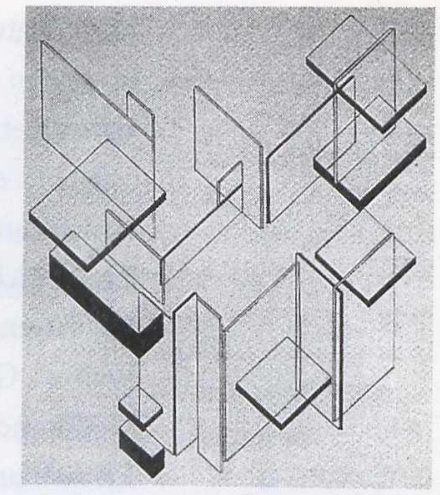

Abb. 12

Man schreibt einen Wettberwerb für ein Rathaus aus, man läßt die

Schüler einer Kunstschule sich um ein Stipendium bewerben, um damit die Erfindungsgabe anzustacheln oder die vielversprechendste Begabung zu entdecken und auf diese Weise das beste Ergebnis zu erzielen. Der ursprüngliche Grund für solche Wettbewerbsformen ist eine solche praktische Absicht jedoch nie geweesen. Im Hintergrund steht immer die uralte Spielfunktion des Wettkampfs als solchen. ${ }^{29}$

Der Idee des Baukastensystems von Gropius und Le Corbusier ging ein entscheidender Schritt voran, der den beiden fortschritts-, technikund daher amerikabegeisterten Architekten nicht entgangen sein dürfte. In seinem Aufsatz The Tall Office Building hatte Louis Sullivan, von Durands Überlegungen ausgehend, das Standardbüro, die Zelle, zur Maßeinheit des gesamten Gebäudes erklärt und damit die Voraussetzung für eine mögliche Kombination von Räumen geschaffen. Sullivan, der Lehrer Frank Lloyd Wrights, verglich das Büro mit der Wabe in einem Bienenstock und legte damit die Basis für Gropius' Wabenbau: „[...] an indefinite number of stories of offices piled tier upon tier, one tier just like another tier, one office just like all other offices - an office being similar to a cell in a honey-comb, merely a compartement, nothing more. “30 Jacques Tati ironisierte in seinem Film Playtime aus dem Jahr 1967 diese bienengleiche Lebensform durch standardisierte, auf einem Raster angeordnete und nach oben offene, quadratische Bürozellen. Die Angestellten agieren nach der ihnen durch die Architektur vorgegebenen Logik, die der Zuschauer zusammen mit M. Hulot neugierig von oben betrachtet. Die rings um das Zentrum, den standard (Telefonzentrale), zurückgelegten Wege ähneln nicht zufällig den Kommunikationsmodellen von Insekten.

Der Maler Theo van Doesburg ging seit etwa 1923 von einem noch grundlegenderen Standpunkt das Problem der modernen Architektur an.
28 Le Corbusier (1928), 106f. Vgl. auch Noell (2002).

29 Huizinga (1987), 188.

30 Sullivan

(1988), 105. Sullivans pädagogisches Interesse ist nicht einem Baukasten, sondern seinen „Kindergarten Chats" von 1901/1902 abzulesen. 
31 Noschka/Knerr

(1986), 34.

32 Zit. in: Unsere liebe Anker-Zeitung (1927), 17. Vgl. auch: Nur ein Spielzeng (1928), 98. Mein Dank für die Auffindung und Bereitstellung dieser Artikel geht an: Leo J. Coffeng vom CVA in den Niederlanden, Dr. Helmut Schwarz, Spielzeugmuseum Nürnberg, und Tobias Mey.
Er zerlegte die Architektur in einzelne Elemente, in Platten, und setzte in seinen Analyse de l'architecture oder Kontra-Konstruktionen genannten axonometrischen Zeichnungen Wände, Decken und Böden gleich. So erreichte er eine Gleichwertigkeit aller räumlichen Bestandteile. In den elementaren Grundlagen der horizontalen und vertikalen Komposition lehnte sich Van Doesburg an Durands Methode an, stellte aber, anders als dieser, den Raum selbst in den Mittelpunkt seiner Überlegungen. Walter Gropius präsentierte auf der Ausstellung des Deutschen Werkbundes auf dem Stuttgarter Weißenhof im Jahr 1927 seinerseits zwei Elementarhäuser. Die zwei Häuser wurden über einem Durandschen/ Fröbelschen Quadratraster entworfen und im Halbtrockenbau sowie im Trockenmontagebau erstellt. Haus 17 sieht man noch auf den Fotografien seine Plattenbauweise an, die 1931 in Kooperation mit den Hirsch Kupfer- und Messingwerken nochmals zur Anwendung kam. In Amerika mündeten die genannten Versuche schließlich in Gropius' und Konrad Wachsmanns berühmtem Packaged House System, dem zerlegbaren und transportablen Fertighaus aus Großplatten.

1927 jährte sich der 75. Todestag Friedrich Fröbels. Aus diesem Grund hatte die Fröbel-Vereinigung schon 1924 den Bau eines Fröbelhauses beschlossen. Der Auftrag zu einem ersten Entwurf ging an das Weimarer Bauhaus und Walter Gropius, der im Sinne der Fröbelschen Lehre Spiel, Leben und Forschung ineinandergreifen lassen wollte. Acht standardisierte Erholungspavillons sollten in Trockenbauweise ausgeführt werden. Walter Gropius wäre mit dem Bau des Fröbel-Hauses eine einzigartige Verschränkung seiner Baugedanken mit deren Grundlagen, dem pädagogischen Spiel, gelungen. In dem zu Ehren Fröbels errichteten Gebäude wäre dann möglicherweise mit dem Bauspiel gespielt worden, das die Bauhaus-Studentin Alma Siedhoff-Buscher im Jahr 1923 zeitgleich mit Gropius' Baukasten im Großen - entworfen hatte. ${ }^{31}$ Geldmangel verhinderte schließlich die Ausführung des Projekts.

\section{Architectus ludens}

Schon in der ersten Nummer der hauseigenen Anker-Zeitung hatte die Redaktion zufrieden festgestellt, daß die Anker-Bausteine zur Anfertigung von Modellbauten „für viele Architekten und Ingenieure unentbehrlich geworden “ seien. ${ }^{32}$ In der Frühlings-Nummer von 1927 hielt man Rückschau auf das bisher Geleistete und dankte den Mitgliedern und Freunden 
für die Mitarbeit; namentlich wurde dem Reichskunstwart Edwin Redslob und dem Bauhaus Dessau unter Walter Gropius gedankt, die sich mehrfach als Preisrichter für einen Bauplan-Wettbewerb zur Verfügung gestellt hatten. In der darauffolgenden Nummer berichtete die Redaktion vom Dessauer Metallhaus, einem Versuchshaus des Bauhausmeisters Georg Muche, und ließ diesen und Walter Gropius zur Standardisierung in der Architektur - nach "Sitte der Dessauer Künstler“ in Kleinschreibung - zu Wort kommen. ${ }^{33}$

Auf dem XXVIII. Plenum der Deutschen Bauakademie 1962 äußerte sich Richard Paulick, ehemaliger Mitarbeiter Muches am Metallhaus, in einem Referat zu den Grundlagen bei der Herausbildung eines „eigenen sozialistischen Stils": Das Baukastensystem sei die Voraussetzung zur "radikalen Standardisierung“ in der Architektur. Der Schweizer Architekt Hans Schmidt, der zwischen 1955 und 1969 in der DDR lebte und arbeitete, unterstrich im anschließenden Artikel die Notwendigkeit eines einheitlichen mathematisch-geometrischen Ordnungssystems, eines Modulsystems und eines Systemliniengitters: „Das Baukastensystem beruht auf der Zerlegung des Bauwerks in einzelne Teile [...].“34 Das Inhaltsverzeichnis des kleinsten Plaspi-Kastens Der kleine Großblock-Baumeister (Typ 1) umfaßte genau 18 Positionen, von der Dachplatte über Wände mit und ohne Fensteröffnung bis zum verschönernden Blumenkasten.

In der Überschreitung der Grenze zwischen normierter und damit auf wenige Bestandteile reduzierter Architektur zu den Kinderspielen sahen manche Kritiker eine unzulässige, „kindische" Vereinfachung der Lebensformen und -qualitäten. Eine Rezension der vom Deutschen Werkbund in Paris 1930 bestrittenen Ausstellung kann dies exemplarisch verdeutlichen. Ein französischer Kritiker fühlte sich angesichts einer von Walter Gropius und seinem Mitarbeiterstab entworfenen und publizierten Treppenkonstruktion aus normierten und vorfabrizierten Metallrosten an den englischen Metallbaukasten Meccano erinnert, der seit 1920 auch in Frankreich hergestellt und vertrieben wurde: „[...] cette construction, qui rappelle un peu celle des mécanos pour enfants. “35

Diese Einschätzung war einerseits durchaus richtig: Während in Frankreich das Idealbild des individuellen Künstlers mit daher ebenfalls individueller Kunst vorherrschte, hatte man sich auf der anderen Seite des Rheins von dieser Auffassung verabschiedet und sah die neue Aufgabe des Künstlers in seiner Vorreiterrolle für eine Reform der Gesellschaft, die in Richtung Standardisierung und Typisierung ging. Es

33 Stablhausbau

(1927), 51-53.

34 Paulick (1962), 169; Schmidt (1962), 170-175.

35 Pawlowski (2002), 325. 

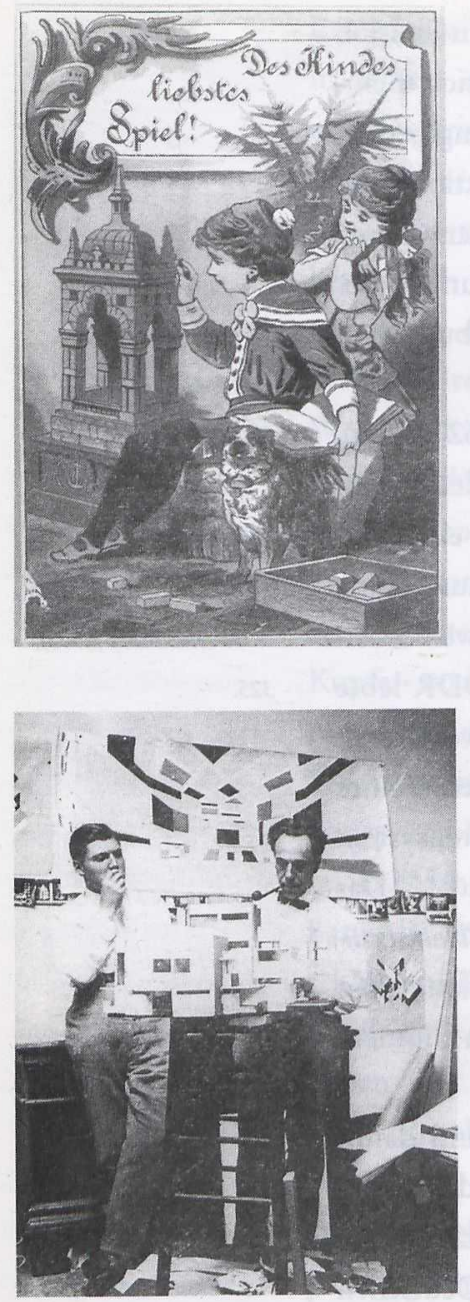

zählte nun nicht mehr der caractère d'art, sondern der valeur' d'usage. ${ }^{36}$ Der von dem Rezensenten ironisch-abwertend gemeinte Vergleich von Kunst mit Spielzeug entsprach vollkommen der Auflösung zweier Grenzen, eben der von Spiel und Kunst und von spielerischen Experimenten und gebrauchsfertiger, reformorientierter Architektur. Diese Auflösung hatte sich von Friedrich Fröbel ausgehend bereits bei dem Lebensreformer Gustav Lilienthal, der zwischen der Entwicklung von Siedlungen, Gartenlauben, Flugapparaten, Kunststickereien und Spielzeug keine klaren Grenzen setzte, mit aller Deutlichkeit angekündigt. Lyonel Feininger negierte mit seiner selbstgeschnitzten und bemalten Stadt am Ende der Welt, die er in seiner Weimarer Bauhauszeit seit 1919 mitsamt ihren Einwohnern realisierte, die klare Trennung von Spiel und Kunst. Feiningers Abbilder von Gelmeroda oder Halle existieren in derFotografie, im Gemälde und im Modellformat. Auch von Le Corbusier hat sich eine Fotografie erhalten, die ihn telefonierend vor einer Spielzeugstadt mit Steildächern zeigt. 1938, also etwa zeitgleich, ist sein Jeu de la Ville Radieuse (JVR) entstanden, ein bis zur Verpackung in der Schachtel ausgearbeiteter Entwurf eines urbanistischen Bauspiels, mit dem man die Kleinstadtidylle auf einem Quadratraster in Richtung einer modernen Idealstadt korrigieren konnte. ${ }^{37}$ Mit Alexander Calders Modellzirkusvorführungen seit 1926, dem Cirque Calder, und seiner fotografischen wie filmischen Dokumentation schien schließlich - Van Doesburg hatte es bereits 1922 postuliert - die Grenze endgültig aufgehoben: „Kunst ist Spiel $[\ldots]$. ${ }^{\text {(338 }}$

36 Pawloreski (2002), 325 .

37 Vgl. Le Corbusier (1983), 485-508. Vgl. Solitaire (1999), 36.

38 Van Doesburg (1984), 174.

Natürlich blieb der grundlegende Unterschied zwischen spielenden Kindern und bauenden Architekten auch weiterhin bestehen. Gemeinsamkeiten sind jedoch auch hier nicht zu übersehen. Der am Modell der Maison Particulière bauende Van Doesburg und der sich nachdenklich gebende Van Eesteren haben ihr Pendant in den Werbeaufnahmen oder Titelabbildungen von Baukästen. Diese zeigen meist ein, häufig auch mehrere Kinder und manchmal sogar Erwachsene beim freudigen und konzentrierten Spiel. Das entstehende Gebäude, sei es ein Denkmal, eine Burg oder ein Einfamilienhaus, steht meist kurz vor der Vollendung durch einen der Porträtierten. Von den anderen wird es bewundernd betrachtet. Für beide Gruppen ist das architektonische Modell experimentelles und 
damit spielerisches Hilfsmittel oder symbolisiert das Ideal einer zukünftigen Gesellschaft. Diese Gemeinsamkeit tritt noch deutlicher zu Tage, wenn man neben den Architekten auch Politiker vor ihren Projektmodellen betrachtet. Hier tritt zum Architekten der Politiker als Entwerfer der Gesellschaft und Visionär hinzu.

Die Parallelen zwischen der Baukunst und dem Baukasten sind vielschichtigerer Art, als daß man sie nur mit der Prägung einer mit den Baukästen aufgewachsenen Architektengeneration erklären oder umgekehrt den Baukasten als einfaches Abbild der bestehenden Welt deuten könnte. Nur auf formale Übereinstimmungen zwischen Baukasten-Architektur und moderner kubischer Architektur hinzuweisen, würde ebenfalls zu kurz greifen. Vielmehr deuten die angeführten Beispiele auf grundsätzlichere Übereinstimmungen hin. Sie

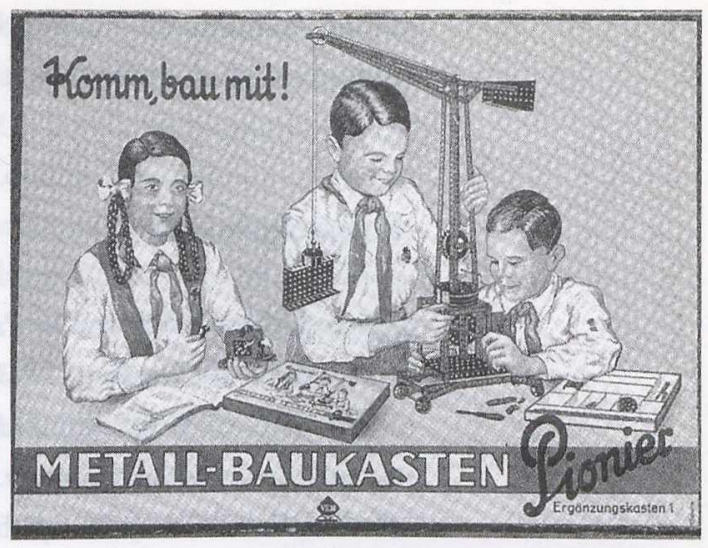
scheinen in einer gemeinsamen Grundlage zu wurzeln, die sowohl bei

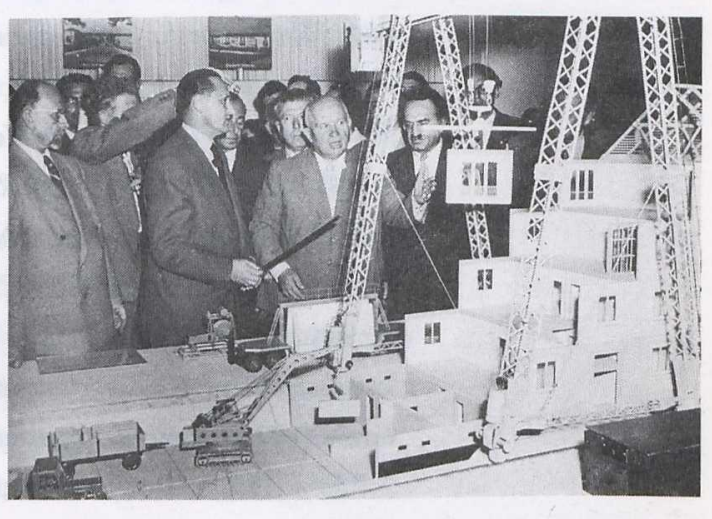
Durand für die Architektur als auch bei Fröbel für das pädagogische Spielzeug greifbar ist: der Suche nach der nicht mehr reduzierbaren Grundform, dem Grundmodul, das ein einheitliches System im Leben und in der Umwelt garantieren kann. Auch für Henry-Russell Hitchcock und Philip Johnson war die Regelmäßigkeit des Rasters, den sie aus dem modernen Skelettbau herleiteten, ein notwendiger Schritt zur Ordnung des Entwurfs und damit der architektonischen Welt. ${ }^{39}$ Wie es aber am besten das Beispiel Le Corbusier zeigt, war die modulare Regelmäßigkeit keine Notwendigkeit, sondern lediglich ein ästhetisches Prinzip, aus dem eine Arbeitsmethode resultierte und das eng mit der formalen Suche nach dem architektonischen Typ verknüpft war.

Für Friedrich Fröbel war hingegen die Suche nach dem Grundbaustein, der Urform der Natur längst abgeschlossen, als er anfing, sich dem Elementarbauspiel zu widmen: „Das Festgestaltete, Kristallinische, ist die erste Erscheinung der irdischen Gestaltung. “40 Seine Nachfolger konnten daher - apodiktischer als selbst die Architekten der Avantgarde der zwanziger

39 Hitchoock/

Johnson (1995), 71.

40 Fröbel (1968); vgl.

Rubin (1989), 24 f.

41 Deckel-Titelblatt

eines Baukastens von

S.F. Fischer aus Ober-

seiffenbach von etwa

1880. Abgebildet in:

Bauspiele (1988), 5. 
Jahre - behaupten: „Für Kinder und Erwachsene. Der Baumeister im Kin dergarten. Nach Fröbel's System: Die Einheit ist der Würfel. ${ }^{\text {*41 }}$

\section{Bibliographie}

Arntz, Helmut (1994): „Der Patenonkel“. In: Wilhelm Kreis. Architekt zweischel Kaiserreich und Demokratie 1873-1955. Hgg.: Winfried Nerdinger u. Eckehard Mai. München, Berlin: Klinkhardt und Biermann, 202-222. Bauspiele (1988): Bauspiele aus zwei Jabrbunderten. Ausstellungskatalog Schweizerisches Museum für Volkskunde. Riehen: Spielzeug- und Dorfmuseum Riehen. Bestelmeier, Georg Hieronimus (1979): Magazin von verschiedenen Kunst- und anderen nützlichen Sachen [...]. ND der Ausgabe Nürnberg 1803 mit einem Vorwort v. Theo Gantner. Zürich: Olms. Cronon, William (1994): „Inconstant Unity: The Passion of Frank Lloyd Wright". In: Frank Lloyd Wright. Architect. Ausstellungskatalog. Museum of Modern Art, New York. Hgg.: Terence Riley/Peter Reed. New York: Museum of Modern Art, 8-31. van Doesburg, Theo (1984): „Der Wille zum Stil“ [1922]. In: De Stijl. Schriften und Manifeste zu einem theoretischen Konzept ästhetischer Umweeltgestaltung. Hgg: Hagen Bächler/Herbert Letsch. Leipzig, Weimar: Gustav Kiepenheuer, 163-179. Durand,Jean-NicolasLouis (1975): Précis des lecons d'architecture, données à l'école royale polytechnique. 2 Bde. ND der 2. überarbeiteten Auflage in 3 Bänden von 1817, 1819 und 1821. Unterschneidheim: Uhl ( $\left.{ }^{1} 1802-1805\right)$. Fröbel, Friedrich (1968): „Die Menschenerziehung [...]“ [1826]. In: Friedrich Fröbel: Ausgewählte Schriften. Bd. 2. Hg.: Erika Hoffmann. Düsseldorf, München: Helmut Küpper. Fröbel, Friedrich (1982): „Einführung der Vierten Gabe [...]“ [1838]. In: Friedrich Fröbel: Ausgewë̈lte Schriften. Bd. 4. Hg.: Erika Hoffmann. Stuttgart: KlettCotta, 89-112. Fröbel, Friedrich (1851): „Anleitung zum rechten Gebrauche der dritten Gabe [...]“ [1851]. In: Friedrich Fröbel: Ausgewwäblte Schriften. Bd. 3. Hg.: Helmut Heiland. Düsseldorf, München: Helmut Küpper, 120-161. Gropius, Walter (1926-27): „der große baukasten“. In: Das Neue Frankfurt 1, 25-30. Hildebrandt, Paul (1979): Das Spielzeng im Leben des Kindes. Reprint Düsseldorf, Köln: Diederichs ( ${ }^{1}$ 1904). Hitchcock, Henry-Russel/Philip Johnson (1995): The International Style. Neuaufl. New York, London: W.W. Norton ( ${ }^{1}$ 1932). Hoek, Els, Hg. (2000): Theo van Doesburg. OEuvre catalogue. Utrecht, Otterloo: Centraal Museum/Kröller-Müller Museum. Huizinga, Johan (1987): Homo Ludens. Vom Ursprung der Kultur im Spiel. Mit einem Nachwort von Andreas Flitner. Hamburg: Rowohlt $\left({ }^{1} 1938\right)$. LebensRäume (1999): LebensRäume und UnternehmensKultur. 100 Jabre Deutsche Werkstätten Hellerau. 
Hgg.: Gabriele Diana Grawe u.a. Hellerau: Deutsche Werkstätten Hellerau. Le Corbusier (1928): „Des yeux qui ne voient pas ... . III. Les autos“. In: Le Corbusier: Vers une Architecture. Paris: Crès, 101-117 ( $\left.{ }^{1} 1923\right)$. Le Corbusier (1983): Buildings and Projects, 1937-1942. New York, London: Garland (= The Le Corbusier Archive 14). Leinweber, Ulf (1999): Baukästen! Technisches Spielzeng vom Biedermeier bis zur Jahrtausendwende. Ausstellungskatalog Staatliche Museen Kassel. Wiesbaden: Drei Lilien (= Schriften zur Volkskunde 7). Lilienthal, Anna/Lilienthal, Gustav (1930): Wege der Technik. Die Lilienthals. Stuttgart, Berlin: Cotta. Manson, Grant (1953): „Wright in the Nursery. The Influence of Froebel Education on the Work of Frank Lloyd Wright”. In: Architectural Review 113/678, 349-352. Nerdinger, Winfried (1996): Walter Gropius. Der Architekt. Berlin: Gebr. Mann ( $\left.{ }^{1} 1986\right)$. Noell, Matthias, u.a., Hgg. (2002): Das Baubaus und Frankreich. Le Bauhaus et la France. Berlin: Akademie (=Passagen. Jahrbuch des Deutschen Forums für Kunstgeschichte 4). Noell, Matthias (2002): „Choisir entre l'individu et le standard.' - Das Künstlerhaus bei Gropius, Le Corbusier, Van Doesburg, Bill.“ In: Noell u.a. (2002), 83-115. Noschka, Anette/Knerr, Günter (1986): Bauklötze staunen. Zweibundert Jabre Geschichte der Baukästen. München: Hirmer. Nur ein Spielzeug (1928): „Nur ein Spielzeug?“ In: Anker-Zeitung 19 (Juli), 98-100. Paulick, Richard (1962): „Die Aufgaben zur Durchsetzung der radikalen Standardisierung als Hauptkettenglied in der gegenwärtigen Etappe des industriellen Bauens“. In: Deutsche Architektur 11, 169. Pawlowski, G. de (2002): „Le Salon des Artistes Décorateurs [...]“. In: La France, 30. Mai, erstes Mal abgedruckt in: Matthias Noell: „Zwischen Krankenhaus und Mönchszelle [...]“. In: Noell u.a. (2002), 325-326. Pettena, Gianni, Hg. (1982): Superstudio 1966-1982. Storie, figure, architettura. Florenz: Electa. Rubin, Jeanne S. (1989): „The Froebel-Wright Kindergarten Connection: A New Perspective“. In: Journal of the Society of Architectural Historians 48 (März), 24-37. Schäche, Wolfgang (1989): „Gustav Lilienthal und das Bauen“. In: Gustav Lilienthal 1849-1933. Baumeister. Lebensreformer, Flugtechniker. Ausstellungskatalog Landesarchiv Berlin. Hg.: Hans J. Reichhardt. Berlin: Stapp, 91-118. Schmidt, Hans (1962): „Baukastensystem und Architektur“. In: Deutsche Architektur 11, 170-175. Solitaire, Marc Ivan Daniel (1999): Le Corbusier: le don de Jeanneret ou les dons de Froebel. Unpubl. Diss. ETH Zürich. Speidel, Manfred, Hg. (1995): Bruno Taut. Natur und Fantasie. Ausstellungskat. Kulturhistorisches Museum Magdeburg. Berlin: Ernst und Sohn. Staatliches Bauhaus Weimar (1923): Staatliches Bauhaus Weimar 1919-1923. Ausstellungskat. Hgg.: Staatliches Bauhaus in Weimar u. Karl Nierendorf. Weimar, München: Bauhaus Verlag. Stahlhausbau (1927): „Stahlhausbau“. In: Anker-Zeitung 16 (Oktober), 51-53. 
Sullivan, Louis (1988): "The Tall Office Building Artistically Considered" [189 In: Louis Sullivan: The Public Papers. Hg.: Robert Twombly. Chicago, London:? University of Chicago Press, 103-113. Suter, Ursula (1993): Hans Schmidt 18 1972. Architekt in Basel, Moskau, Berlin-Ost. Zürich: gta. Szambien, Werner (198Jean-Nicolas-Louis Durand 1760-1834. De l'imitation à la norme. Paris: Picard. Tatu Manfredo, Hg. (1981): Five Architects N.Y. Rom: Officina Edizioni ( $\left.{ }^{1} 1976\right)$. Bruno (1919): Alpine Architektur. Hagen: Folkwang. Ueber die zweckmäß39g Methode zu projectiren (1863): „Ueber die zweckmäßigste Methode zu projer ren“. In: Zeitschrift für praktische Baukunst 23, 101-104. Unsere liebe Anker-Zeitw (1927): „Unsere liebe Anker-Zeitung“. In: Anker-Zeitung 14 (April), 7-21. Wh' Iain Boyd/Schneider, Romana, Hgg. (1986): Die Briefe der Gläsernen Kette. Bet Ernst. Wright, Frank Lloyd (1966): Ein Testament. Zur neuen Architektur. Münch Langen und Müller/Rowohlt.

\section{Abbildungen}

Abb. 1: Superstudio: Möbelserie „Misura“, um 1969-1971. In: Pettena (1982), 73. Abb. 2: Jean-Nicolas-Louis Durand: Grundriß. In: Durand (1975), Bd. 1, 2. Teil, 2, Fig. 2.

Abb. 3: Friedrich Fröbel: Schönheitsformen. In: Fröbel (1974), 160.

Abb. 4: Jean-Nicolas-Louis Durand: Teilung eines Quadrates. In: Durand (1975), 1, 2. Teil, Taf. 20.

Abb. 5: Friedrich Fröbel: Schönheitsformen. In: Fröbel (1974), 160.

Abb. 6: Friedrich Fröbel: Teilung des Würfels (Ausschnitt). In: Fröbel (1974), 158. Abb. 7: Peter Eisenman: House III, Entwurfssystem (Ausschnitt), 1969-1971. Tafuri (1981), 47.

Abb. 8-9: Gustav Lilienthal: Kinderhaus, o.D. u. Terrasthaus, Fotografie, um 1908. Reichhardt (1989), 84 u. 114.

Abb. 10: Walter Gropius: Wabenbau, 1922. In: Noell u.a. (2002), 88.

Abb. 11: Le Corbusier: Maison standardisée (Ausschnitt), 1925. In: Noell u.a. (200 89.

Abb. 12: Theo van Doesburg: Kontrakonstruktion, 1923. In: Noell u.a. (2002), 88 Abb. 13: Richters Anker-Steinbaukasten, Anzeige, um 1890. In: Leinweber (19\% 64.

Abb. 14: Theo van Doesburg/Cornelis van Eesteren: Fotografie, 1923. In: H (2000), 342.

Abb. 15: Werbefotografie Baukasten Pionier, um 1960. In: Leinweber (1999), 121. Abb. 16: Walter Ulbricht/Gerhard Kosel: Nikita S. Chruschtschow auf der Berlit Bauausstellung 1957. In: Suter (1993), 87. 